\title{
Acknowledgment to Reviewers of Journal of Functional Biomaterials in 2021
}

\author{
Journal of Functional Biomaterials Editorial Office
}

Citation: Journal of Functional

Biomaterials Editorial Office.

Acknowledgment to Reviewers of Journal of Functional Biomaterials in 2021. J. Funct. Biomater. 2022, 13, 10 https://doi.org/10.3390/jfb13010010

Published: 26 January 2022

Publisher's Note: MDPI stays neutral with regard to jurisdictional claims in published maps and institutional affiliations.

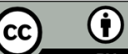

Copyright: $\odot 2022$ by the author. Licensee MDPI, Basel, Switzerland. This article is an open access article distributed under the terms and conditions of the Creative Commons Attribution (CC BY) license (https://creativecommons.org/licenses/by/4.0/).
MDPI AG, St. Alban-Anlage 66, 4052 Basel, Switzerland

Rigorous peer-reviews are the basis of high-quality academic publishing. Thanks to the great efforts of our reviewers, Journal of Functional Biomaterials was able to maintain its standards for the high quality of its published papers. Thanks to the contribution of our reviewers, in 2021, the median time to first decision was 18 days and the median time to publication was 46 days. The editors would like to extend their gratitude and recognition to the following reviewers for their precious time and dedication, regardless of whether the papers they reviewed were finally published:

$\begin{array}{ll}\text { Abanteriba, Sylvester } & \text { Diogo, Patricia } \\ \text { Abbas Shojaosadati, Seyed } & \text { Dioguardi, Mario } \\ \text { Ali, Lamiaa } & \text { Dragoman, Daniela } \\ \text { Alves, Gutemberg } & \text { Drukteinis, Saulius } \\ \text { Apostu, Dragos } & \text { Dudas, Zoltan } \\ \text { Baltatu, Madalina } & \text { Dudea, Diana } \\ \text { Banciu, Cristina } & \text { Dudziec, Beata } \\ \text { Barbaro, Katia } & \text { Dupin, Damien } \\ \text { Beregoi, Mihaela } & \text { Esposito, Antonella } \\ \text { Berteau, Jean-Philippe } & \text { Falkowski, Paweł } \\ \text { Brzeszczynska, Joanna } & \text { Fareed, Muhammad } \\ \text { Byrne, Hugh } & \text { Farina, Ilenia } \\ \text { Cali, Michele } & \text { Franca, Rodrigo } \\ \text { Carata, Elisabetta } & \text { Fu, Wenxin } \\ \text { Carvalho, Marta } & \text { Gadomska-Gajadhur, Agnieszka } \\ \text { Cerrone, Federico } & \text { Gentile, Gennaro } \\ \text { Cervino, Gabriele } & \text { Geurtsen, Werner } \\ \text { Chaudhry, Rasul } & \text { Ghanbari, Siavash } \\ \text { Chen, Min-Hua } & \text { Gheorghiu, Mihaela } \\ \text { Chen, Yingchieh } & \text { Giacomo, Viviana } \\ \text { Chen, Yupeng } & \text { Girjob, Claudia } \\ \text { Cheng, Huanyu } & \text { Gloria, Antonio } \\ \text { Chiulan, Ioana } & \text { Gorseta, Kristina } \\ \text { Chlanda, Adrian } & \text { Grassia, Vincenzo } \\ \text { Chrcanovic, Bruno } & \text { Grocholewicz, Katarzyna } \\ \text { Cianci, Pasquale } & \text { Gurikov, Pavel } \\ \text { Cid, Emili } & \text { Haidar, Ziyad } \\ \text { Cifuentes, Sandra } & \text { Han, Woo-Suck } \\ \text { Csoka, Levente } & \text { Hotta, Harumi } \\ \text { Dabaghi, Mohammadhossein } & \\ \text { Dash, Biraja } & \text { Itel, Fabian } \\ \text { D'Errico, Gerardino } & \text { Jartinez, Juan } \\ & \\ \text { Chameep }\end{array}$


Jain, Gaurav

Jeong, Gunjae

Jiang, Can

Kalluru, Poliraju

Kasiotis, Kostas

Kawai, Toshiyuki

Kharmanda, Ghais

Kheirollahi Bisheh, Hossein

Kim, Bongju

Kim, Sang

Konstantinos, Katakalos

Kozakiewicz, Marcin

Kundanati, Lakshminath

Lacina, Lukas

Lai, Wen-Fu

Lee, Chia-Hung

Li, Xiangjia

Lieder, Marek

List, Manuela

Lovecchio, Joseph

Luca, Dorin

Lucio, Maria

Mao, Hongli

Marin, Ricardo

Marsili, Enrico

Marto, Carlos

Maturavongsadit, Panita

Mavelli, Fabio

Mazur, Marta

Miculescu, Florin

Mlynarczyk, Dariusz

Mondanelli, Nicola

Morariu, Simona

Nasr, Maha

Nautiyal, Amit

Nica, Cristina

Nikolaivits, Efstratios

Olivier, Florian

Orchel, Arkadiusz

Ortiz De Zarate, David

Ozkan, Gokcekaya

Padmanabhan, Sanosh

Palka, Lukasz

Pall, Emoke

Parfenova, Lyudmila

Parsonage, Derek

Patching, Simon

Patel, Dinesh

Peditto, Matteo

Petrenko, Alexander

Pieniak, Daniel
Pilmane, Mara

Piras, Paolo

Pirzada, Tahira

Pixley, Sarah

Poling, Mikaela

Prasadh, Somasundaram

Preti, Giulio

Prochon, Miroslawa

Pyriochou, Anastasia

Qin, Liguo

Rosenkranz, Andreas

Roy, Dhruvajyoti

Rutkowska, Maria

Rybka, Jakub

Salaun, Fabien

San Miguel, Veronica

Sandquist, David

Sandu, Andrei

Sanjairaj, Vijayavenkataraman

Sanna, Stefano

Sapudom, Jiranuwat

Saravanakumar, Kandasamy

Sarecka-Hujar, Beata

Sawicki, Jacek

Schiavi, Jessica

Seok, Hyun

Shi, Xianai

Signore, Giovanni

Sikder, Prabaha

Siodmiak, Jacek

Smiga-Matuszowicz, Monika

Spinato, Sergio

Spriano, Silvia

Stachewicz, Urszula

Stan, Miruna

Sterzynski, Tomasz

Stewart, Todd

Sun, Bo

Szarek, Arkadiusz

Tan, Hsih-Yin

Tischer, Paula

Tomasevic, Igor

Tsai, Shiao-Wen

Ullah, Imran

Ungor, Ditta

velasco ortega, eugenio

Verron, Elise

Vijayan, Vineeth

Vingolo, Enzo

Visnapuu, Triinu

W. Nicholson, John 
Wang, Jeffrey

Wang, Ning

Winiecki, Mariusz

$\mathrm{Wu}$, Haigang

Xiong, Xin

Yamada, Yusuke

Yan, Yanling

Ye, Lin

$\mathrm{Yu}$, Le

Zaharia, Catalin
Zhang, Jibo

Zhang, Lianbin

Zhang, Shuye

Zhang, Wei

Zhang, Xing

Zhang, Yanhu

Zhao, Liqing

Zheng, Mengmeng

Zhu, Lei 